\title{
Ultrasound-induced opening of the blood-brain barrier to enhance temozolomide and irinotecan delivery: an experimental study in rabbits
}

\author{
Kevin Beccaria, MD, ${ }^{1,2}$ Michael Canney, PhD, ${ }^{1}$ Lauriane Goldwirt, PharmD, PhD, ${ }^{3}$ \\ Christine Fernandez, PharmD, PhD, ${ }^{3}$ Julie Piquet, MS, ${ }^{4}$ Marie-Cécile Perier, MSc, ${ }^{5}$ \\ Cyril Lafon, $\mathrm{PhD},{ }^{6}$ Jean-Yves Chapelon, $\mathrm{PhD},{ }^{6}$ and Alexandre Carpentier, MD, $\mathrm{PhD} 7,8$
}

\begin{abstract}
${ }^{1}$ CarThera Research Team, Brain and Spine Institute, Paris; ${ }^{2}$ Department of Neurosurgery, Assistance Publique-Hopitaux de Paris, Necker Enfants Malades Hospital, Paris; ${ }^{3}$ Clinical Pharmacy Department, College of Pharmacy, Paris Sud University, Chatenay Malabry; ${ }^{4}$ Laboratory of Biosurgical Research, University of Paris Descartes, Sorbonne Paris Cite, Paris; ${ }^{5 I N S E R M ~}$ U970, Centre de recherche cardiovasculaire de Paris-Epidémiologie cardiovasculaire, Paris; ${ }^{6}$ INSERM U1032, LabTau, University of Lyon; ${ }^{7}$ Assistance Publique-Hopitaux de Paris, Pitie Salpetriere Hospital, Department of Neurosurgery, Paris; and ${ }^{8}$ Paris 6 Sorbonne University, School of Medicine, Paris, France
\end{abstract}

OBJECTIVE The blood-brain barrier (BBB) limits the intracerebral penetration of drugs and brain tumor treatment efficacy. The effect of ultrasound-induced BBB opening on the intracerebral concentration of temozolomide (TMZ) and irinotecan (CPT-11) was assessed.

METHODS This study was performed using 34 healthy New Zealand rabbits. Half had unilateral BBB opening, and half served as controls. Sonications were performed by pulsing a $1.05-\mathrm{MHz}$ planar ultrasound transducer with a duty cycle of $2.5 \%$ and an in situ acoustic pressure level of $0.6 \mathrm{MPa}$ after injection of a microbubble ultrasound contrast agent. Drugs were injected either 5 minutes before (ChemoPreUS) or 15 minutes after (ChemoPostUS) the ultrasound sonication. The plasma and intracerebral concentrations of both drugs were quantified using ultra-performance liquid chromatography.

RESULTS The mean intracerebral tissue-to-plasma drug concentration ratio in the control hemispheres was $34 \%$ for TMZ and $2 \%$ for CPT-11. After BBB opening, these values increased by up to $21 \%$ for TMZ and up to $178 \%$ for CPT- 11 . Intracerebral concentrations of drugs were enhanced in regions where the BBB was opened compared with the contralateral hemisphere $(p<0.01$ and $p<0.0001$ for CPT-11, $p=0.02$ and $p=0.03$ for TMZ, in ChemoPreUS and ChemoPostUS, respectively) and compared with the control group ( $p<0.001$ and $p<0.0001$ for CPT-11, $p<0.01$ and $p=0.02$ for TMZ, in ChemoPreUS and ChemoPostUS, respectively). The intracerebral distribution of drugs was heterogeneous, depending on the distance from the ultrasound source.

CONCLUSIONS Ultrasound-induced opening of the BBB significantly enhances the intracerebral concentration of both TMZ and CPT-11 in rabbits.

http://thejns.org/doi/abs/10.3171/2015.4.JNS142893

KEY WORDS blood-brain barrier; brain tumors; drug delivery; irinotecan; oncology; temozolomide; ultrasound

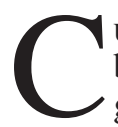

URRENTLY, the prognosis for patients with primary brain tumors, despite aggressive treatment strategies, remains poor. The median survival for patients with high-grade gliomas varies from 1 to 5 years. ${ }^{26}$ The failure of the current standard of treatment is due to a combination of incomplete resection of the malignant tissue during surgery (infiltrative disease) and a low penetration of drugs not only into the tumor, but also into the surrounding brain parenchyma, where up to $80 \%$ of recurrences happen..$^{12,35}$ The low penetration of drugs into the brain is due to the existence of the blood-brain barrier (BBB), which consists of endothelial cells with tight junctions that line the microvasculature of the brain. ${ }^{8}$ This physiological barrier limits approximately $98 \%$ of smallmolecule drugs and $100 \%$ of large-molecule drugs from reaching the parenchymal tissue. ${ }^{22}$

ABBREVIATIONS BBB = blood-brain barrier; BCNU = 1,3-bis(2-chloroethyl)-1-nitrosourea; $C P T-11$ = irinotecan; IV = intravenous; MGMT = 0-6-methylguanine-DNA methyltransferase; TMZ = temozolomide; $\mathrm{UCA}=$ ultrasound contrast agent.

SUBMITTED December 20, 2014. ACCEPTED April 7, 2015.

INCLUDE WHEN CITING Published online November 13, 2015; DOI: 10.3171/2015.4.JNS142893. 
Although several methods have been proposed to increase the permeability of the BBB, ${ }^{6,21,39}$ pulsed ultrasound is one that has shown significant promise in laboratory studies conducted over the past decade. Using a pulsed ultrasound source in combination with an ultrasound contrast agent (UCA), the application of low-power sonications on brain parenchyma can safely and temporarily open the BBB for durations of 6-8 hours. ${ }^{14,34,36}$ Mechanisms of opening the BBB by pulsed ultrasound may include transcytosis, transendothelial opening with cell fenestrations, and opening of tight junctions. ${ }^{27}$ All of these mechanisms may potentially allow for increased passage of drug molecules.

An improved uptake of drugs into the brain after ultrasound-induced BBB disruption has been demonstrated for antineoplastic drugs, such as doxorubicin; ;,24,30,31,41 1,3bis(2-chloroethyl)-1-nitrosourea (BCNU); ;8,29 temozolomide (TMZ) ${ }^{38}$ and cisplatin; ${ }^{40}$ in addition to high-molecular-weight antibodies, such as trastuzumab, ${ }^{23}$ dopamine receptor antibodies, ${ }^{17}$ and anti- $\beta$-amyloid antibodies. ${ }^{16}$ Increased drug delivery to the brain is associated with few side effects, and studies in rat glioma models have shown that tumor control and increased survival can be

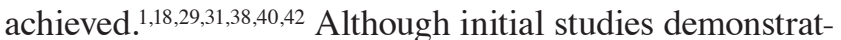
ing safe BBB opening by pulsed ultrasound were performed in small-animal models, recent studies have confirmed these results in nonhuman primates. ${ }^{19,20}$

The human skull represents the principle obstacle to the use of ultrasound in neurooncology, because ultrasound energy is attenuated and distorted by the skull bone. While transcranial ultrasound delivery into the brain is in clinical development for certain indications, the technique requires MRI monitoring, complicated electronics, and corrections for phase distortion and attenuation. ${ }^{5,13}$ Therefore, the technique may not be suited for repetitive BBB opening every month, which is required to optimize intravenous chemotherapy delivery for diffuse gliomas. Considering the fact that surgery is systematically performed for the treatment of primary brain tumors, either to resect the tumor (debulking surgery) or to remove a tissue sample for histological analysis (biopsy), a new implantable ultrasound device has been developed to take advantage of the skull bone opening. By implanting a small ultrasound device into the bone during these surgical procedures, distortion of the ultrasound energy can be avoided, and the procedure can be simplified. In a recent publication by our team, this concept was demonstrated, and a planar 1 $\mathrm{MHz}$ unfocused ultrasound transducer was used to open the BBB in a large but targeted volume in healthy rabbit brain. ${ }^{2}$ The BBB disruption was visualized using Evans blue dye and confirmed using 4.7-T MRI after intravenous injection of $\mathrm{Gd}$. The opening was limited to the extent of the ultrasound beam, and at acoustic pressure levels below $0.8 \mathrm{MPa}$, side effects were limited to a few perivascular red blood cell extravasations.

The present work was performed to assess the influence of ultrasound-induced BBB opening by an unfocused ultrasound transducer on the concentration of drugs in the brain parenchyma. Irinotecan (CPT-11) and TMZ were chosen, because both drugs are used clinically in the treatment of brain tumors.

\section{Methods \\ Preparation of Animals}

All of the animal experiments were approved by a local ethics committee (Comité d'Ethique en matière d'Expérimentation Animale, Paris-Descartes, France). This study was performed using 34 healthy male New Zealand white rabbits. Animals were anesthetized with an intramuscular injection of a mixture of xylazine $(9 \mathrm{mg} /$ $\mathrm{kg}$; Rompun, 2\% solution, Bayer) and ketamine (50 mg/ $\mathrm{kg}$; Imalgène, solution of $1000 \mathrm{mg} / 10 \mathrm{ml}$, Merial). Additional injections were performed during the experiments if necessary. A femoral vein was catheterized for perfusion of isotonic saline, drugs, and UCA. Analgesia was ensured with $1.5 \mathrm{ml}$ of lidocaine (Xylocaine 1\%, scalp infiltration) and intravenous (IV) bolus of morphine (Morphine Lavoisier, $10 \mathrm{mg} / \mathrm{ml}$ ). A bur hole craniectomy was unilaterally performed from the coronal suture to $12 \mathrm{~mm}$ posteriorly. Euthanization of the animals was performed by IV injection of pentobarbital sodium salt $(54.7 \mathrm{mg} / 100$ $\mathrm{ml})$. Brains were extracted at the end of each experiment and immediately frozen $\left(-20^{\circ} \mathrm{C}\right)$. A blood sample was harvested 5 minutes before each animal was humanely killed. Plasma was extracted from the blood sample by centrifugation and frozen $\left(-20^{\circ} \mathrm{C}\right)$.

\section{Ultrasound Experimental Setup}

A custom-built, air-backed, 1-MHz single-element transducer was constructed by using a $10-\mathrm{mm}$ flat piezoceramic disc (Pz 26, Ferroperm Piezoceramics). The transducer was operated at a center frequency of $1.05 \mathrm{MHz}$ and a pulse repetition frequency of $1 \mathrm{~Hz}$, with a pulse length of 25,000 cycles $(2.5 \%$ duty cycle, $23.2 \mathrm{msec}$ total burst duration) and an in situ acoustic pressure level of 0.6 MPa. These parameters correspond to safe parameters for BBB opening that were determined in a previous study. ${ }^{2}$ For each session of BBB opening, a bolus injection of microbubbles (Sonovue, $0.1 \mathrm{ml} / \mathrm{kg}$, Bracco Imaging) was injected through a peripheral vein followed by a $2-\mathrm{ml}$ flush of saline. Sonovue is a UCA made of sulfur hexafluoride gas enveloped in a phospholipid shell with a mean diameter of $2.5 \mu \mathrm{m}$. The total sonication duration was 2 minutes. Microbubbles were injected at the same time as the start of the ultrasound sonication. Additional details about the device, monitoring of BBB opening using Evans blue dye diffusion, and MRI are described in a previous publication. ${ }^{2}$

\section{Drug Injection Protocols}

For both drugs, 2 experimental protocols were used in this study (Fig. 1). In each protocol, the time of reference (T0) corresponded to the beginning of the ultrasound sonication in the presence of the UCA. In the first protocol (ChemoPreUS, $\mathrm{n}=16$ ), the drug was injected 5 minutes before sonication (T-5 min). Blood samples were taken at 25 minutes $(\mathrm{T}+25 \mathrm{~min})$, and animal euthanization was performed at 30 minutes $(\mathrm{T}+30 \mathrm{~min})$ after sonication. In the second protocol (ChemoPostUS, $n=18$ ), the drug was injected 15 minutes after sonication $(\mathrm{T}+15 \mathrm{~min})$. Blood samples were taken at 40 minutes ( $\mathrm{T}+40 \mathrm{~min}$ ), and animal euthanization was performed at 45 minutes ( $\mathrm{T}+45$ 

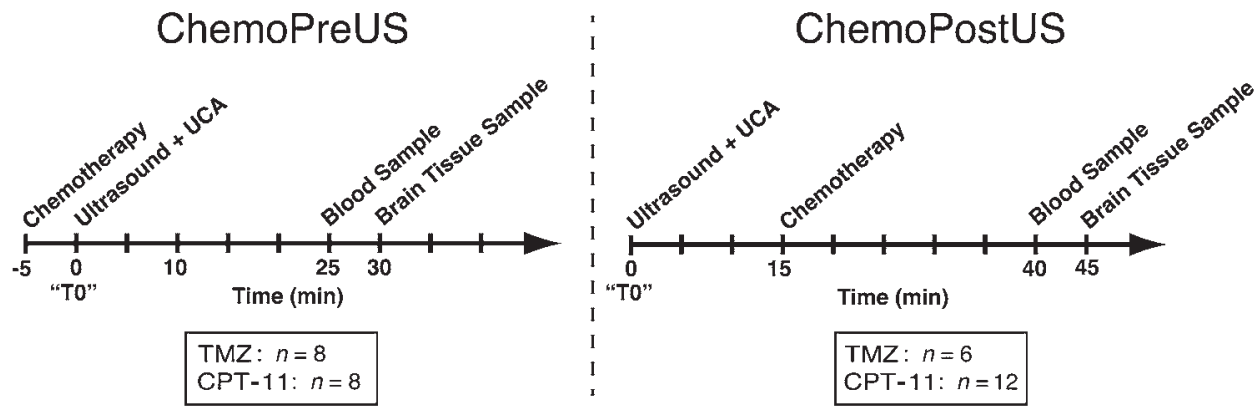

FIG. 1. Experimental protocols used in this study. In Protocol 1 (ChemoPreUS, left), chemotherapy was administered 5 minutes before the start of ultrasound sonication, while in protocol 2 (ChemoPostUS, right), chemotherapy was administered 15 minutes after ultrasound was applied. Note that the time of exposure to the drug $(30 \mathrm{~min})$ was the same in both protocols.

min) after sonication. In each protocol, the drug circulated for 30 minutes after BBB opening. Considering the halflives of TMZ and CPT-11, and taking into account the fact that the brain/plasma concentration ratio of both drugs is stable during their distribution, ${ }^{9}$ this delay was adapted to highlight any drug enhancement. Moreover, it corresponded to a previously published protocol. ${ }^{29,38}$

CPT-11 was injected intravenously at a dose of $6 \mathrm{mg} / \mathrm{kg}$ (Irinotecan Ebewe Pharma France, Sandoz $40 \mathrm{mg} / 2 \mathrm{ml}$ ) and TMZ at a mean dose of $4.7 \mathrm{mg} / \mathrm{kg}$, corresponding to a dose of $75 \mathrm{mg} / \mathrm{m}^{2}$ of body surface $\left(\right.$ in $\left.\mathrm{m}^{2}\right)$, with $\mathrm{S}=(\mathrm{K}$ $\left.\times \mathrm{P}^{2 / 3}\right) / 10^{4}$, in which $\mathrm{P}$ represents the weight of the animal and $\mathrm{K}=9.5$. These doses corresponded to doses used in clinical practice..$^{28,33}$

For each experimental protocol group, half of the rabbits $(n=17)$ underwent sonication on a region of the right hemisphere after craniotomy and in the presence of Sonovue. The other half $(n=17)$ served as controls and received only a right bur hole craniotomy, without sonication and without Sonovue injection.

\section{Quantification of TMZ and CPT-11}

A slice of brain parenchyma, centered along the acoustic axis of the ultrasound transducer, was cut from each rabbit brain. Four representative samples $(450 \mathrm{mg}$ each) were extracted from each slice: a cortical and a deep brain sample from each hemisphere (Fig. 2). A number was attributed to each sample for further blinded analysis. Chromatography was performed on an Acquity UPLC system (Waters Corp.) with an autosampler temperature of $6^{\circ} \mathrm{C}$. Separation was performed at $25^{\circ} \mathrm{C}$ on a Waters Acquity UPLC BEH C18 column $(2.1 \mathrm{~mm} \times 100 \mathrm{~mm}, 1.7-\mu \mathrm{m}$ particle size) with a linear gradient elution using water (containing $0.1 \%$ formic acid and $10 \mathrm{mM}$ ammonium acetate) and acetonitrile as the mobile phase.

TMZ and CPT-11 quantification in plasma and brain was performed following previously published methods using ultra-performance liquid chromatography with tandem mass spectrometry detection..$^{10,11}$

\section{Statistical Analysis}

Statistical analysis was performed using the R Project (version 3.0.2). Parenchyma concentrations were systematically divided by the corresponding plasma concentra- tion to obtain a ratio of concentration, expressed as a percentage. All comparisons were performed with the concentration ratios. Data are summarized using mean (SD) values. Comparisons of drug concentration ratios between the group that underwent sonication and the control group were performed using the Student t-test. Within each group, the diffusion ratio between sonicated hemispheres and nonsonicated hemispheres were compared using the paired Student t-test. For all analyses, a 2-tailed $\mathrm{p}$ value < 0.05 was considered statistically significant.

\section{Results}

This study was performed using a total of 34 rabbits. Fourteen rabbits received TMZ, 8 in the ChemoPreUS protocol and 6 in the ChemoPostUS protocol. Twenty rabbits received CPT-11, 8 in the ChemoPreUS protocol and 12 in the ChemoPostUS protocol. Mean plasma and brain parenchyma concentrations and corresponding concentration ratios for both protocols are reported in Table 1.

\section{Ultrasound-Induced Opening of the BBB and Drug Delivery to the Brain}

For TMZ, the mean concentration ratio (brain/plasma) in sonicated hemispheres was significantly higher than the mean concentration ratio in nonsonicated contralateral hemispheres (Fig. 3 upper), for both the ChemoPreUS (41.4\% vs $34.7 \%$, respectively; $\mathrm{p}<0.001)$ and Chemo-
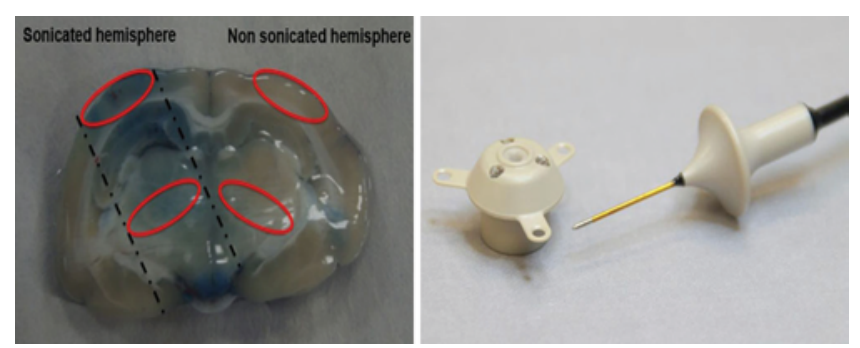

FIG. 2. Left: Results of ultrasound-induced BBB opening from our previous work. ${ }^{2}$ The ultrasound field (represented by the black dashed lines) where the BBB is opened is colored in blue (from Evan's blue dye). $\mathrm{Pa}$ renchyma samples were taken from the cortex and from the deep brain on both sides of the brain (red circles). Right: Prototype of the unfocused ultrasound transducer and its transcutaneous connecting system. 
TABLE 1. Mean plasma and brain parenchyma concentrations and corresponding concentration ratios for ChemoPreUS and ChemoPostUS protocols*

\begin{tabular}{|c|c|c|c|c|c|c|c|}
\hline \multirow[b]{2}{*}{ Protocol } & \multirow[b]{2}{*}{ Group } & \multicolumn{3}{|c|}{ TMZ Concentration $(n=14)$} & \multicolumn{3}{|c|}{ CPT-11 Concentration $(n=20)$} \\
\hline & & $\begin{array}{l}\text { Plasma } \\
\text { (ng/ml) }\end{array}$ & Brain $(n g / g)$ & $\begin{array}{c}\text { Mean Ratio Brain/ } \\
\text { Plasma (\%) }\end{array}$ & $\begin{array}{l}\text { Plasma } \\
\text { (ng/ml) }\end{array}$ & Brain (ng/g) & $\begin{array}{c}\text { Mean Ratio Brain/ } \\
\text { Plasma (\%) }\end{array}$ \\
\hline \multirow[t]{13}{*}{ ChemoPreUS $(n=16)$} & Sonicated rabbits & \multirow{7}{*}{$3999 \pm 628$} & & & \multirow{7}{*}{$3366 \pm 426$} & & \\
\hline & US hemisphere & & & & & & \\
\hline & Cortex & & $1785 \pm 141$ & 45 & & $238 \pm 82$ & 7 \\
\hline & Deep brain & & $1493 \pm 255$ & 37 & & $162 \pm 31$ & 5 \\
\hline & Non-US hemisphere & & & & & & \\
\hline & Cortex & & $1421 \pm 260$ & 36 & & $83 \pm 8$ & 2 \\
\hline & Deep brain & & $1357 \pm 261$ & 34 & & $112 \pm 14$ & 3 \\
\hline & Nonsonicated controls $†$ & \multirow{6}{*}{$4135 \pm 242$} & & & \multirow{6}{*}{$3163 \pm 140$} & & \\
\hline & Non-US hemisphere & & & & & & \\
\hline & Cortex & & $1457 \pm 101$ & 37 & & $68 \pm 13$ & 2 \\
\hline & Deep brain & & $1274 \pm 121$ & 31 & & $60 \pm 12$ & 2 \\
\hline & Cortex & & $1575 \pm 97$ & 38 & & $75 \pm 15$ & 2 \\
\hline & Deep brain & & $1239 \pm 97$ & 30 & & $70 \pm 17$ & 2 \\
\hline \multirow[t]{13}{*}{ ChemoPostUS $(n=18)$} & Sonicated rabbits & \multirow{7}{*}{$3426 \pm 179$} & & & \multirow{7}{*}{$3357 \pm 257$} & & \\
\hline & US hemisphere & & & & & & \\
\hline & Cortex & & $1626 \pm 88$ & 48 & & $229 \pm 67$ & 7 \\
\hline & Deep brain & & $1320 \pm 63$ & 39 & & $226 \pm 66$ & 7 \\
\hline & Non-US hemisphere & & & & & & \\
\hline & Cortex & & $1331 \pm 115$ & 39 & & $54 \pm 16$ & 2 \\
\hline & Deep brain & & $1197 \pm 72$ & 35 & & $83 \pm 27$ & 2 \\
\hline & Nonsonicated controls $†$ & \multirow{6}{*}{$3947 \pm 213$} & & & \multirow{6}{*}{$3171 \pm 526$} & & \\
\hline & Non-US hemisphere & & & & & & \\
\hline & Cortex & & $1373 \pm 132$ & 35 & & $89 \pm 40$ & 3 \\
\hline & Deep brain & & $1305 \pm 127$ & 33 & & $90 \pm 51$ & 3 \\
\hline & Cortex & & $1652 \pm 217$ & 42 & & $88 \pm 55$ & 3 \\
\hline & Deep brain & & $1216 \pm 60$ & 31 & & $90 \pm 38$ & 3 \\
\hline
\end{tabular}

US = ultrasound

* Values given as mean \pm SD unless otherwise indicated.

$\dagger$ Nonsonicated control rabbits have 2 hemispheres; therefore deep brain and cortex are listed twice.

PostUS (43.1\% vs $36.9 \%$, respectively; $\mathrm{p}=0.03)$ protocols. This significant difference was also confirmed when the sonicated hemispheres of rabbits that had undergone sonication were compared with the same hemispheres in control rabbits that received no ultrasound, for both the ChemoPreUS (41.4\% vs $33 \%$ respectively; $\mathrm{p}<0.01)$ and ChemoPostUS ( $43.1 \%$ vs $34.1 \%$ respectively; $\mathrm{p}=0.02$ ) protocols.

For CPT-11, the mean concentration ratio (brain/plasma) in sonicated hemispheres was significantly higher than the mean concentration ratio in nonsonicated contralateral hemispheres (Fig. 3 lower), for both the ChemoPreUS (6.1\% vs $2.9 \%$, respectively; $\mathrm{p}=0.02)$ and ChemoPostUS (6.8\% vs $2.1 \%$, respectively; $\mathrm{p}<0.0001$ ) protocols. This significant difference was also confirmed when the sonicated hemispheres of rabbits that underwent sonication were compared with the same hemispheres in control rabbits that received no ultrasound, for both the ChemoPreUS $(6.1 \%$ vs $2 \%$, respectively; $\mathrm{p}<0.001)$ and
ChemoPostUS (6.8\% vs $2.8 \%$, respectively; $\mathrm{p}<0.0001$ ) protocols.

\section{Concentration Enhancement of TMZ in the Brain and Depth of Sonication}

For TMZ, the mean concentration ratio within the cortex varied from $36 \%$ to $38 \%$ in nonsonicated cortex and was $45 \%$ in sonicated cortex of rabbits that underwent sonication in the ChemoPreUS protocol $(p=0.04$, comparison between both hemispheres in rabbits that underwent sonication; $\mathrm{p}=0.02$, comparison between sonicated cortex of rabbits that underwent sonication and nonsonicated cortex of control rabbits). The mean concentration ratios varied from $35 \%$ to $42 \%$ in nonsonicated cortex and was $48 \%$ in sonicated cortex in the ChemoPostUS protocol (p $=0.07$, comparison between both hemispheres in rabbits that underwent sonication; $\mathrm{p}=0.03$, comparison between sonicated cortex of rabbits that underwent sonication and nonsonicated cortex of control rabbits). In the deep brain 

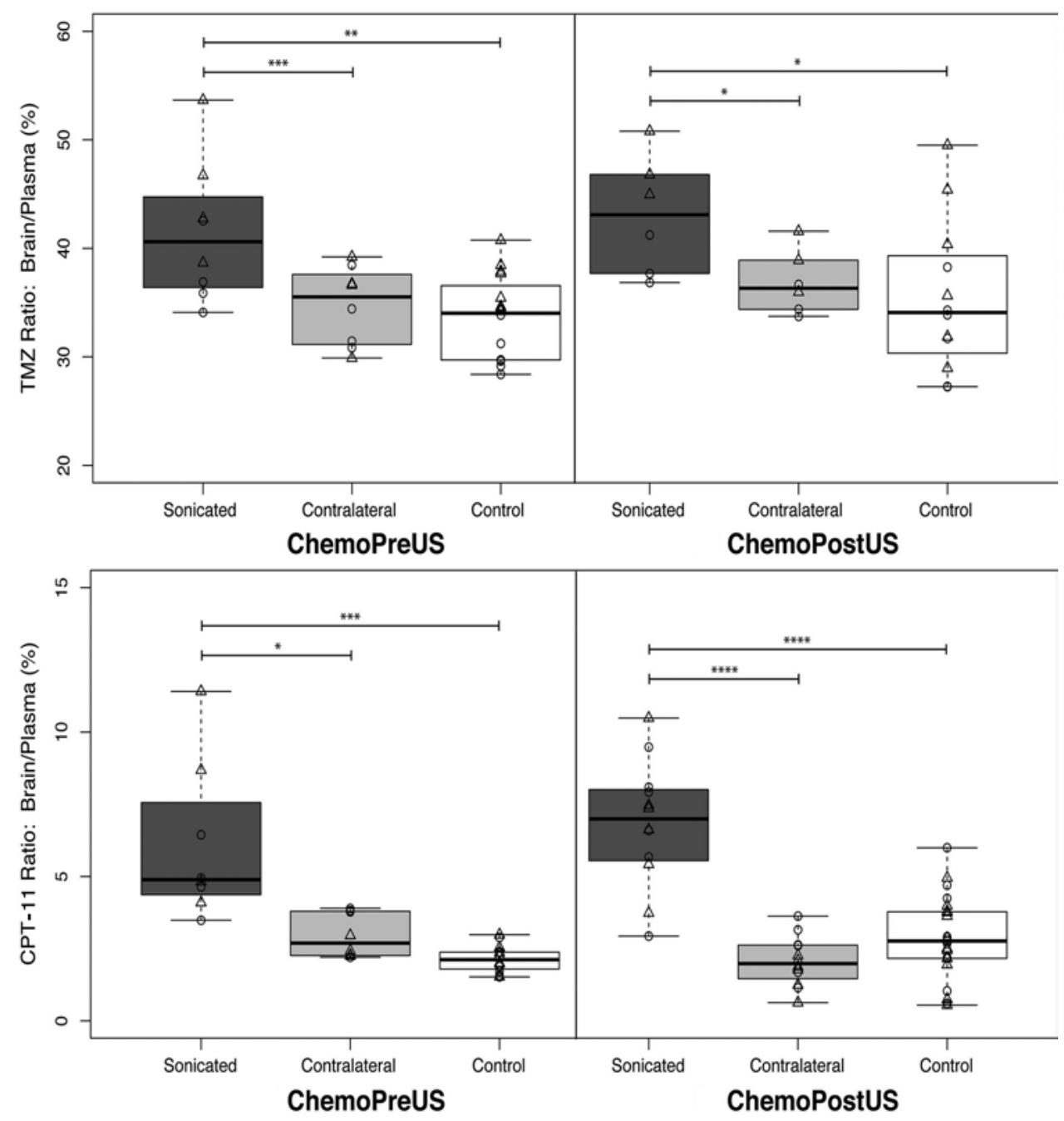

FIG. 3. TMZ (upper) and CPT-11 (lower) concentration ratios (brain/plasma) for the ChemoPreUS (left) and ChemoPostUS (right) protocols. Contralateral $=$ nonsonicated hemispheres of rabbits that underwent sonication. Control $=$ both hemispheres of rabbits that did not undergo sonication. Sonicated $=$ sonicated hemispheres of rabbits that underwent sonication. Data points for the cortex (triangles) and deep brain (circles) as well as statistical significance are indicated $\left({ }^{*} p<0.05,{ }^{* *} p<0.01,{ }^{* * *} p<0.001,{ }^{* * * *} p\right.$ $<0.0001)$.

parenchyma, the TMZ concentration ratio of the rabbit group that underwent sonication was statistically different between sonicated and nonsonicated contralateral deep brain parenchyma only for the ChemoPreUS protocol $(\mathrm{p}$ $=0.01)$ and not for the ChemoPostUS protocol $(\mathrm{p}=0.15)$. When comparing the TMZ concentration of sonicated deep brain parenchyma with rabbits that did not undergo sonication (controls), a statistically significant difference appeared in ChemoPreUS (37\% vs $31 \%$, respectively; $p=$ $0.02)$, which was not observed in the ChemoPostUS (39\% vs $33 \%$, respectively; $\mathrm{p}=0.2$ ).

For CPT-11, no significant statistical difference was observed in concentration ratios when sonicated and nonsonicated cortex of rabbits that underwent sonication were compared in the ChemoPreUS protocol $(7 \%$ versus $2 \%$, respectively, $p=0.06$ ). In all other cases, the mean concentration ratios were significantly higher in both sonicated cortex and deep brain parenchyma in both ChemoPreUS and ChemoPostUS protocols. The mean ratio was $7 \%$ in sonicated cortex while varying from $2 \%$ to $3 \%$ in nonsonicated cortex in both ChemoPreUS and ChemoPostUS. The mean ratio varied from $5 \%$ to $7 \%$ in sonicated deep brain parenchyma, while it varied from $2 \%$ to $3 \%$ in nonsonicated deep brain parenchyma in both ChemoPreUS and ChemoPostUS.

\section{Reliability of the Experimental Model}

No statistical differences were observed in the concentration of TMZ and CPT-11 when comparing both hemispheres of rabbits that did not undergo sonication (controls). This observation was true for both cortex and deep brain parenchyma, in both ChemoPreUS and ChemoPostUS. No difference was observed either between nonsonicated contralateral hemispheres of rabbits that underwent sonication and hemispheres of rabbits that did not (controls). Ratios of concentration varied from $30 \%$ to 
$42 \%$ for TMZ and from $2 \%$ to $3 \%$ for CPT- 11 . One exception was observed when penetration of TMZ was compared in the cortex of nonsonicated hemispheres of rabbits that underwent sonication with those that did not (controls) for ChemoPreUS $(\mathrm{p}=0.02)$.

\section{Comparison Between Mean Enhancement of CPT-11 and Mean Enhancement of TMZ After Sonication}

In protocol ChemoPreUS, the mean concentration ratio of TMZ was $34 \%$ in the brain parenchyma of control rabbits, $35 \%$ in the nonsonicated hemispheres of rabbits that underwent sonication, and $41 \%$ in sonicated hemispheres of rabbits that underwent sonication. In protocol ChemoPostUS, mean values were $35 \%, 37 \%$, and $43 \%$, respectively. Thus, the mean delivery enhancement of TMZ after sonication was $21 \%$ and $19 \%$ in ChemoPreUS and ChemoPostUS protocols, respectively.

In protocol ChemoPreUS, the mean concentration ratio of CPT-11 was $2 \%$ in brain parenchyma of control rabbits, $2.5 \%$ in the nonsonicated hemispheres of rabbits that underwent sonication, and $6 \%$ in sonicated hemispheres of rabbits that underwent sonication. In protocol ChemoPostUS, mean values were $3 \%, 2 \%$, and $7 \%$, respectively. Thus, the mean delivery enhancement of CPT-11 after sonication was $178 \%$ and $162 \%$ in ChemoPreUS and ChemoPostUS protocols, respectively.

The mean enhancement ratio was not significantly different between ChemoPreUs and ChemoPostUS for both CPT-11 $(\mathrm{p}=0.50)$ and TMZ $(\mathrm{p}=0.62)$.

\section{Discussion}

Contrary to many previous studies published in the literature that use external focused ultrasound devices, the implantable unfocused ultrasound device currently being developed-and which has an identical acoustic field to the version used in these studies-is particularly adapted to be easy to use for repetitive clinical treatments. The device is designed to be implanted in a $12-\mathrm{mm}$ skull bur hole, a size that is commonly used during craniotomies, either immediately following resection of the tumor or during a unique surgical procedure. The device is activated on demand during repeated chemotherapy sessions by connecting a transcutaneous needle that has been developed to plug into the implant. Activation is performed in a procedure that lasts approximately 15 minutes and which a skilled nurse can perform. In addition, the device has been designed to be compatible with MRI, which allows for monitoring of treatment follow-up.

The use of unfocused ultrasound allows for sonication of a large volume of brain parenchyma, corresponding to the size of brain tumors or surgical cavities after tumor removal. It was observed in a previous publication that opening of the BBB could be performed in regions where the in situ acoustic pressure was less than half that of the acoustic pressure applied along the acoustic axis. This corresponded to volumes of almost $2 \mathrm{~cm}^{3}(2.5-\mathrm{cm}$ high cylinder with a radius of $0.5 \mathrm{~cm}$ ) with a transducer of $1 \mathrm{~cm}$ in diameter. ${ }^{2}$ In contrast, focused ultrasound systems have a small focal volume $(3 \times 6 \mathrm{~mm})$ and therefore must be electronically steered or mechanically scanned to disrupt the BBB in larger volumes. For example, McDannold et al. used a focused transcranial ultrasound system and performed sonications over 9 treatment points with a spacing of $2 \mathrm{~mm}$ to disrupt the BBB in a volume of tissue of $1 \mathrm{~cm}^{3}$. Scanning of the focus over a larger volume using such devices is possible, but potentially increases the procedure time and leads to additional uncertainty in the acoustic pressure at the focus. ${ }^{20}$

While there have been no previous studies on the diffusion of CPT-11 after ultrasound-induced opening of the $\mathrm{BBB}$, Wei et al..$^{38}$ recently assessed the therapeutic effect of TMZ in association with ultrasound-induced opening of the BBB on rats implanted with 9L glioma cells. In their study, they observed that the TMZ CSF/plasma ratio was increased from $22.7 \%$ to $32.6 \%$ due to ultrasound exposures, while the 7-day tumor progression ratio was reduced from 24 to 5 days and median survival was extended from 20 to 23 days.

The mean concentration ratio observed in the current study (varying from $30 \%$ to $42 \%$, Table 1) was on the same order of magnitude as the ratio reported by Wei et al. (CSF/plasma ratio of 22.7\%). ${ }^{38}$ The ratio observed in this study of 3\% for CPT-11 was significantly lower. These results imply that both drugs spontaneously can pass the intact BBB but with a very low rate, and with a better passage for TMZ than for CPT-11. This is in accordance with the partial and transient efficiency of these drugs on brain tumors and the better results obtained with TMZ than with CPT-11.28,33 In addition, both clinical and experimental observations confirm that the use of high doses of both TMZ and CPT-11 increase their antitumoral activity. ${ }^{3,4,15,25,37}$ For both TMZ and CPT-11, the main limit to high-dose protocols remains the systemic side effects of these drugs. In this context, ultrasound-induced opening of the BBB appears to be an ideal technique that may enhance drug delivery to the brain without having to increase the amount of systemically administered drug.

Penetration of drug was heterogeneous when taking into account the depth of sonication in the brain parenchyma. While the few number of cases studied in this work may partially explain such a result, this observation is also in accordance with previously published studies in which opening of the BBB was more intense in the gray matter than in the white matter. ${ }^{2,20}$ It has been hypothesized that this difference is due to a more dense vasculature network in the cortex (gray matter) than in the deep brain parenchyma (mix of white and gray matter), ${ }^{7}$ causing the surface of exchange between blood circulation and brain parenchyma to be higher in the gray matter. This will need to be taken into consideration in future clinical applications.

No significant differences were observed between concentration ratios in the ChemoPreUS and ChemoPostUS protocols, so that the time of injection, before or after sonication, did not have any influence on drug passage through the BBB. The consequence in clinical practice is that the time of injection can be adapted to drugs, depending on their pharmacological and pharmacodynamic characteristics. Injection may be performed before the sonication if the direct effect of ultrasound on the drug may increase local delivery, for example with encapsulated drugs that would be liberated when entering the ultrasound field. ${ }^{29}$ 
Alternatively, in the case of delicate molecules, injection after sonication may be better, because it avoids the mechanical effects of ultrasound on the drug molecule.

While enhanced tumor control was not shown in our study, many others have shown that enhanced penetration of chemotherapeutics after BBB opening can have significant effects on tumor growth and survival in animal models. For example, doxorubicin delivery was studied by Treat et al. who were able to enhance the doxorubicin concentration in rat brain up to therapeutic levels..$^{30}$ This corresponded to a 3.5-fold enhancement (about 252\% enhancement), and it was associated with minimal vascular effects and no macroscopic tissue damage. Even further enhancement was also possible (up to 23.5-fold the control concentration) but was associated with severe tissue damage and neuronal death. The same authors showed that enhanced delivery of liposomal doxorubicin reduced tumor growth and increased survival in rats inoculated with gliosarcoma cells in the brain. ${ }^{31}$ Liu et al.$^{18}$ also demonstrated antitumoral effects of ultrasound-induced BBB opening with $\mathrm{BCNU}$ on rats implanted with $\mathrm{C} 6$ glioma cells. Treatments resulted in control of tumor progression and significantly increased animal survival compared with rats treated by ultrasound or BCNU alone. Tumor control and increased survival were also observed in studies using BCNU encapsulated in microbubbles. ${ }^{29}$ The pegylated capsules used in the study not only protected BCNU from rapid hydrolysis, but also reduced drug exposure and toxicity to other normal tissues, particularly the liver.

Both clinical and experimental observations have confirmed that the use of high doses of both TMZ and CPT-11 increase their antitumoral activity. On one hand, removal of O-6-methylguanine by the DNA repair protein O-6-methylguanine-DNA methyltransferase (MGMT) promotes resistance to TMZ in human glioma cell lines, ${ }^{3}$ but it has been hypothesized that high doses of TMZ may induce MGMT saturation and overcome this resistance. ${ }^{37}$ Such a saturation of MGMT would explain the longer survival obtained with protracted and continuous schedules in humans ${ }^{25}$ or with increased local brain delivery of TMZ by biodegradable polymers in a rodent glioma model. ${ }^{4} \mathrm{On}$ the other hand, Jiang et al. have recently shown that high doses of CPT-11 may increase the in vivo inhibition of U87 tumor growth in nude mice models. ${ }^{15}$

In the same way, some studies suggest that a modest enhancement of either TMZ or CPT-11 may be sufficient to obtain a significant improvement in their antitumoral activity. The $170 \%$ increase in CPT-11 brain distribution after BBB opening that was observed in our study is clinically relevant considering previous studies. Verreault et al. showed that using a liposomal formulation of CPT-11 provided a $25 \%$ increase in CPT-11 in the brain and led to a significant increase in average survival (63.1 vs 58.3 days, after liposomal and normal formulation administration, respectively). ${ }^{32}$ It has also been demonstrated that TMZ and CPT-11 are drug efflux transporter ABCB1 (P-glycoprotein) substrates that have been associated with higher TMZ and CPT-11 brain distribution in ABCB1-deficient mice. ${ }^{9}$ Interestingly, the amount of increase in brain-toblood ratios obtained after BBB opening (1.37-fold and 2.70-fold higher after ultrasound for TMZ and CPT-11, respectively) were close to those obtained in ABCB1deficient mice (1.1 and 2.1 for TMZ and CPT-11, respectively), suggesting that $\mathrm{BBB}$ opening reverses the effects of $\mathrm{ABCB} 1$ on drug brain distribution, which is of great interest in brain tumor treatment.

\section{Conclusions}

The BBB constitutes a major impediment to drug delivery to the brain in the treatment of primitive brain tumors, and it is now well known that low-power pulsed ultrasound can open the BBB in a safe and reversible manner. In the present work, it has been demonstrated that both TMZ and CPT-11 delivery into the brain parenchyma could be enhanced after opening of the BBB by an unfocused ultrasound device in the presence of microbubble agents. By using a transducer that can be plugged into the skull at the end of a regular procedure (biopsy/debulking), such a technique may allow oncologists to repeatedly and transiently open the BBB at the time of drug administration. Drug delivery to the brain and tumoral tissue could be improved while systemic doses could be decreased to reduce side effects and the subsequent morbidity of chemotherapy.

\section{Acknowledgments}

CarThera and the Paris Regional Innovation Center supported this work. We gratefully acknowledge Alain Birer, Adrien Matias, and Alexandre Vignot for help with the design and construction of transducers and the experimental setup. We also gratefully acknowledge all members of the team of the Laboratory of Biosurgical Research, Paris, France, and its director Professor Philippe Menasché, for their help in the realization of animal experiments.

\section{References}

1. Aryal M, Vykhodtseva N, Zhang YZ, Park J, McDannold N: Multiple treatments with liposomal doxorubicin and ultrasound-induced disruption of blood-tumor and blood-brain barriers improve outcomes in a rat glioma model. J Control Release 169:103-111, 2013

2. Beccaria K, Canney M, Goldwirt L, Fernandez C, Adam $\mathrm{C}$, Piquet J, et al: Opening of the blood-brain barrier with an unfocused ultrasound device in rabbits. J Neurosurg 119:887-898, 2013

3. Bobola MS, Tseng SH, Blank A, Berger MS, Silber JR: Role of O6-methylguanine-DNA methyltransferase in resistance of human brain tumor cell lines to the clinically relevant methylating agents temozolomide and streptozotocin. Clin Cancer Res 2:735-741, 1996

4. Brem S, Tyler B, Li K, Pradilla G, Legnani F, Caplan J, et al: Local delivery of temozolomide by biodegradable polymers is superior to oral administration in a rodent glioma model. Cancer Chemother Pharmacol 60:643-650, 2007

5. Clement GT, Sun J, Giesecke T, Hynynen K: A hemisphere array for non-invasive ultrasound brain therapy and surgery. Phys Med Biol 45:3707-3719, 2000

6. Doolittle ND, Miner ME, Hall WA, Siegal T, Jerome E, Osztie E, et al: Safety and efficacy of a multicenter study using intraarterial chemotherapy in conjunction with osmotic opening of the blood-brain barrier for the treatment of patients with malignant brain tumors. Cancer 88:637-647, 2000

7. Duvernoy HM, Delon S, Vannson JL: Cortical blood vessels of the human brain. Brain Res Bull 7:519-579, 1981

8. Fortin D: [The blood-brain barrier should not be underesti- 
mated in neuro-oncology.] Rev Neurol (Paris) 160:523-532, 2004 (Fr)

9. Goldwirt L, Beccaria K, Carpentier A, Farinotti R, Fernandez C: Irinotecan and temozolomide brain distribution: a focus on ABCB1. Cancer Chemother Pharmacol 74:185-193, 2014

10. Goldwirt L, Lemaitre F, Zahr N, Farinotti R, Fernandez C: A new UPLC-MS/MS method for the determination of irinotecan and 7-ethyl-10-hydroxycamptothecin ( $\mathrm{SN}-38)$ in mice: application to plasma and brain pharmacokinetics. J Pharm Biomed Anal 66:325-333, 2012

11. Goldwirt L, Zahr N, Farinotti R, Fernandez C: Development of a new UPLC-MSMS method for the determination of temozolomide in mice: application to plasma pharmacokinetics and brain distribution study. Biomed Chromatogr 27:889893, 2013

12. Hochberg FH, Pruitt A: Assumptions in the radiotherapy of glioblastoma. Neurology 30:907-911, 1980

13. Hynynen K, Jolesz FA: Demonstration of potential noninvasive ultrasound brain therapy through an intact skull. Ultrasound Med Biol 24:275-283, 1998

14. Hynynen K, McDannold N, Vykhodtseva N, Jolesz FA: Noninvasive MR imaging-guided focal opening of the bloodbrain barrier in rabbits. Radiology 220:640-646, 2001

15. Jiang P, Mukthavaram R, Chao Y, Bharati IS, Fogal V, Pastorino S, et al: Novel anti-glioblastoma agents and therapeutic combinations identified from a collection of FDA approved drugs. J Transl Med 12:13, 2014 (Erratum in J Transl Med 12:126, 2014)

16. Jordão JF, Ayala-Grosso CA, Markham K, Huang Y, Chopra $\mathrm{R}$, McLaurin J, et al: Antibodies targeted to the brain with image-guided focused ultrasound reduces amyloid-beta plaque load in the TgCRND8 mouse model of Alzheimer's disease. PLoS One 5:e10549, 2010

17. Kinoshita M, McDannold N, Jolesz FA, Hynynen K: Targeted delivery of antibodies through the blood-brain barrier by MRI-guided focused ultrasound. Biochem Biophys Res Commun 340:1085-1090, 2006

18. Liu HL, Hua MY, Chen PY, Chu PC, Pan CH, Yang HW, et al: Blood-brain barrier disruption with focused ultrasound enhances delivery of chemotherapeutic drugs for glioblastoma treatment. Radiology 255:415-425, 2010

19. Marquet F, Tung YS, Teichert T, Ferrera VP, Konofagou EE: Noninvasive, transient and selective blood-brain barrier opening in non-human primates in vivo. PLoS One 6:e22598, 2011

20. McDannold N, Arvanitis CD, Vykhodtseva N, Livingstone MS: Temporary disruption of the blood-brain barrier by use of ultrasound and microbubbles: safety and efficacy evaluation in rhesus macaques. Cancer Res 72:3652-3663, 2012

21. Neuwelt EA, Howieson J, Frenkel EP, Specht HD, Weigel R, Buchan CG, et al: Therapeutic efficacy of multiagent chemotherapy with drug delivery enhancement by blood-brain barrier modification in glioblastoma. Neurosurgery 19:573582, 1986

22. Pardridge WM: Blood-brain barrier delivery. Drug Discov Today 12:54-61, 2007

23. Park EJ, Zhang YZ, Vykhodtseva N, McDannold N: Ultrasound-mediated blood-brain/blood-tumor barrier disruption improves outcomes with trastuzumab in a breast cancer brain metastasis model. J Control Release 163:277-284, 2012

24. Park J, Zhang Y, Vykhodtseva N, Jolesz FA, McDannold NJ: The kinetics of blood brain barrier permeability and targeted doxorubicin delivery into brain induced by focused ultrasound. J Control Release 162:134-142, 2012

25. Pouratian N, Gasco J, Sherman JH, Shaffrey ME, Schiff D: Toxicity and efficacy of protracted low dose temozolomide for the treatment of low grade gliomas. J Neurooncol 82:281-288, 2007
26. Sanai N, Berger MS: Extent of resection influences outcomes for patients with gliomas. Rev Neurol (Paris) 167:648-654, 2011

27. Sheikov N, McDannold N, Vykhodtseva N, Jolesz F, Hynynen $\mathrm{K}$ : Cellular mechanisms of the blood-brain barrier opening induced by ultrasound in presence of microbubbles. Ultrasound Med Biol 30:979-989, 2004

28. Stupp R, Mason WP, van den Bent MJ, Weller M, Fisher B, Taphoorn MJ, et al: Radiotherapy plus concomitant and adjuvant temozolomide for glioblastoma. $\mathbf{N}$ Engl J Med 352:987-996, 2005

29. Ting CY, Fan CH, Liu HL, Huang CY, Hsieh HY, Yen TC, et al: Concurrent blood-brain barrier opening and local drug delivery using drug-carrying microbubbles and focused ultrasound for brain glioma treatment. Biomaterials 33:704-712, 2012

30. Treat LH, McDannold N, Vykhodtseva N, Zhang Y, Tam K, Hynynen K: Targeted delivery of doxorubicin to the rat brain at therapeutic levels using MRI-guided focused ultrasound. Int J Cancer 121:901-907, 2007

31. Treat LH, McDannold N, Zhang Y, Vykhodtseva N, Hynynen $\mathrm{K}$ : Improved anti-tumor effect of liposomal doxorubicin after targeted blood-brain barrier disruption by MRI-guided focused ultrasound in rat glioma. Ultrasound Med Biol 38:1716-1725, 2012

32. Verreault M, Strutt D, Masin D, Anantha M, Waterhouse D, Yapp DT, et al: Irinophore $C^{\mathrm{TM}}$, a lipid-based nanoparticulate formulation of irinotecan, is more effective than free irinotecan when used to treat an orthotopic glioblastoma model. J Control Release 158:34-43, 2012

33. Vredenburgh JJ, Desjardins A, Reardon DA, Friedman HS: Experience with irinotecan for the treatment of malignant glioma. Neuro Oncol 11:80-91, 2009

34. Vykhodtseva NI, Hynynen K, Damianou C: Histologic effects of high intensity pulsed ultrasound exposure with subharmonic emission in rabbit brain in vivo. Ultrasound Med Biol 21:969-979, 1995

35. Wallner KE, Galicich JH, Krol G, Arbit E, Malkin MG: Patterns of failure following treatment for glioblastoma multiforme and anaplastic astrocytoma. Int J Radiat Oncol Biol Phys 16:1405-1409, 1989

36. Wang F, Cheng Y, Mei J, Song Y, Yang YQ, Liu Y, et al: Focused ultrasound microbubble destruction-mediated changes in blood-brain barrier permeability assessed by contrastenhanced magnetic resonance imaging. J Ultrasound Med 28:1501-1509, 2009

37. Wang ZJ, Altonok D, Sood S, Konski A, Poulik J: Inadvertent high-dose therapy with temozolomide in a child with recurrent pontine glioma followed by a rapid clinical response but deteriorated after substitution with low-dose therapy. J Pediatr Hematol Oncol 36:e549-e552, 2014

38. Wei KC, Chu PC, Wang HYJ, Huang CY, Chen PY, Tsai HC, et al: Focused ultrasound-induced blood-brain barrier opening to enhance temozolomide delivery for glioblastoma treatment: a preclinical study. PLoS One 8: 58995, 2013

39. Williams PC, Henner WD, Roman-Goldstein S, Dahlborg SA, Brummett RE, Tableman M, et al: Toxicity and efficacy of carboplatin and etoposide in conjunction with disruption of the blood-brain tumor barrier in the treatment of intracranial neoplasms. Neurosurgery 37:17-28, 1995

40. Yang FY, Horng SC: Chemotherapy of glioblastoma by targeted liposomal platinum compounds with focused ultrasound. Conf Proc Annu Int Conf IEEE Eng Med Biol Soc 2013:6289-6292, 2013

41. Yang FY, Wang HE, Liu RS, Teng MC, Li JJ, Lu M, et al: Pharmacokinetic analysis of 111 in-labeled liposomal Doxorubicin in murine glioblastoma after blood-brain barrier disruption by focused ultrasound. PLoS One 7:e45468, 2012

42. Yang FY, Wong TT, Teng MC, Liu RS, Lu M, Liang HF, et 
al: Focused ultrasound and interleukin-4 receptor-targeted liposomal doxorubicin for enhanced targeted drug delivery and antitumor effect in glioblastoma multiforme. J Control Release 160:652-658, 2012

\section{Disclosures}

Alexandre Carpentier, Kevin Beccaria, Michael Canney, Cyril Lafon, and Jean-Yves Chapelon have submitted a patent application on this technology and licensed it to CarThera. Alexandre Carpentier, Michael Canney, Cyril Lafon, and Jean-Yves Chapelon have ownership in CarThera SAS, a spin-off start-up company from Paris VI Sorbonne University. Michael Canney is an employee of CarThera SAS. Kevin Beccaria's research year was supported by CarThera SAS.

\section{Author Contributions}

Conception and design: Carpentier, Beccaria, Canney, Fernandez, Lafon, Chapelon. Acquisition of data: Beccaria, Canney, Goldwirt, Piquet. Analysis and interpretation of data: Carpentier,
Beccaria, Canney, Goldwirt, Fernandez, Lafon, Chapelon. Drafting the article: Carpentier. Critically revising the article: Carpentier, Beccaria, Canney, Goldwirt, Fernandez, Lafon, Chapelon. Reviewed submitted version of manuscript: Carpentier, Beccaria, Canney. Approved the final version of the manuscript on behalf of all authors: Carpentier. Statistical analysis: Beccaria, Canney, Perier. Study supervision: Carpentier.

\section{Supplemental Information}

Previous Presentation

Portions of this work were presented in poster form at the IEEEUFFC Symposium, Prague, Czech Republic, July 25, 2013, and as an oral presentation at the 13th International Symposium on Therapeutic Ultrasound, May 13, 2013.

\section{Correspondence}

Alexandre Carpentier, Department of Neurosurgery, Pitie-Salpetriere Hospital, Assistance Publique Hôpitaux de Paris, Sorbonne University, Paris 6 School of Medicine, Paris 75013, France. email: alexandre.carpentier@psl.aphp.fr. 